\title{
Equilibrio dinámico y calidad del movimiento en corredores aficionados
}

\author{
Dynamic balance and quality of the movement in amateur runner
}

* Nicolás Gómez-Álvarez, * Ernesto Mora Jiménez, * Brian Astorga Cáceres, * Neiel Contreras Quintanilla, * Ignacio Cancino Vásquez, * Gustavo Pavez-Adasme

Gómez, N., Mora, E., Astorga, B., Contreras, N., Cancino, I., Pavez, G. (2019). Equilibrio dinámico y calidad del movimiento en corredores aficionados. Revista Ciencias de la Actividad Física UCM, N 20(1), enero-junio, 1-11. DOI: http://doi.org/10.29035/rcaf.20.1.8

\section{RESUMEN}

El objetivo de este estudio es describir el equilibrio dinámico y control neuromuscular asociado a riesgo de lesión en corredores recreativos adultos de la comuna de Chillán. Metodología: El estudio presenta un diseño no experimental, transeccional descriptivo. La muestra corresponde a 61 corredores aficionados entre 18 y 56 años de edad, 39 hombres $(63,9 \%)$ y 22 mujeres $(36,1 \%)$ elegidos por criterios de inclusión y exclusión. Los instrumentos utilizados fueron los test Y Balance Test (YBT) y Lateral Step Down (LSD) Resultados. La comparación entre grupos etarios, tanto de los resultados del YBT y LSD no reportaron diferencias significativas $(p>0,05)$. Conclusión. Los corredores recreativos tienen una moderada calidad de movimiento y un equilibrio dinámico similar a la población adulta sana, sin embargo, la asimetría presentada en el alcance $(>4 \mathrm{~cm})$ es considerado un factor de riesgo para lesiones en extremidad inferior.

\section{PALABRAS CLAVE}

Corredores, lesiones en extremidad inferior, prueba de reducción lateral, prueba de balance en Y.

\begin{abstract}
The objective of this study is to describe the dynamic balance and neuromuscular control associated with injury risk in adult runners of the Chillan community. Methodology: The study presents a nonexperimental, descriptive transactional design. The sample corresponds to 61 amateur runners between 18 and 56 years of age, 39 men (63.9\%) and 22 women (36.1\%) chosen by inclusion and exclusion criteria. The instruments used were the Y Balance Test (YBT) and the Lateral Step Down (LSD) test. Results: The comparison between age groups of both YBT and LSD results did not report significant differences ( $p>0.05)$. Conclusion: The recreational runners have a moderate movement quality and a dynamic balance similar to the healthy adult population, however, the asymmetry presented in the range $(>4 \mathrm{~cm})$ is considered a risk factor for injuries in the lower extremity.
\end{abstract}

\section{Key words}

Runners, lower extremity injuries, lateral reduction test, $\mathrm{Y}$ balance test.

* Universidad Adventista de Chile. Chillán, Chile. 


\section{INTRODUCCIÓN}

La inactividad física y la obesidad se han convertido mundialmente en los principales factores de riesgo de mortalidad prematura (Guthold, Stevens, Riley, \& Bull, 2018; Nyberg, Batty, Pentti, Virtanen, Alfredsson, Fransson et al., 2018). Ambos factores de riesgo son modificables a través de la práctica regular de actividad física (AF) considerando que aquellas personas que presentan un mejor fitness cardiorrespiratorio y muscular tienen una menor posibilidad de morbimortalidad prematura (Clausen, Marott, Holtermann, Gyntelberg, \& Jensen, 2018), por lo que intervenciones preventivas que incluyan aumentar la actividad física son ampliamente recomendadas (Crump, Sundquist, Winkleby, \& Sundquist, 2017). Se estima que en Chile el $31,8 \%$ de la población realiza algún tipo de actividad física, siendo el running una de las disciplinas con más adeptos a nivel nacional (Sandoval Vilches \& García Pacheco, 2014).

El aumento en los últimos años de esta actividad ha estado favorecido por un incremento exponencial de instancias que promocionan las carreras recreativas e incentivan a que personas comiencen a participar de estos eventos deportivos, que además son consideradas de bajo. Sin embargo, hay que tener en cuenta que la falta de gradación y la inserción temprana a la competencia contribuyen al desarrollo de lesiones deportivas como micro traumatismos reiterados o por sobrecarga debido al trabajo excesivo sobre articulaciones, músculos y tendones de personas que se inician en un deporte (de Araujo, Baeza, Zalada, Alves, \& de Mattos, 2015). Estudios han señalado que la falta de experiencia de corredores aficionados o novatos tiende a ser uno de los principales factores de riesgo para desarrollar alguna lesión, causando una interrupción o abandono de la actividad física futura (Buist, Bredeweg, Lemmink, Pepping, Zwerver, Van Mechelen et al., 2007), las cuales pueden alcanzar hasta un $84,9 \%$ en aquellos que lo hacen de manera recreacional y localizándose principalmente en la extremidad inferior (Kluitenberg, Van Middelkoop, Diercks, \& Van der Worp, 2015). En este contexto se hace fundamental la incorporación estrategias que permitan prevenir le- siones y disminuir la deserción temprana de la práctica deportiva.

Los instrumentos de evaluación pre-participativos (EPP) parecen tener un potencial para identificar la población en riesgo de lesión. La Asociación Nacional de Entrenadores Atléticos (NSCA), propone dentro de la EPP realizar un examen físico que integre entre otras cosas un análisis de la función musculo-esquelética, de tal manera que pueda encontrar anomalías como: asimetría en el volumen muscular, déficit en el rango articular, debilidad muscular o cualquier otra deformidad obvia que permitan generar un programa de acondicionamiento para las zonas problemáticas entre 4 a 6 semanas previo al inicio de entrenamiento (Conley, Bolin, Carek, Konin, Neal \& Violette, 2014). En este contexto las pruebas funcionales permiten identificar déficit en el control de movimiento o movimientos limitados que aumentan el riesgo de lesión en deportistas (Brumitt, Heiderscheit, Manske, Niemuth, Mattocks \& Rauh, 2018; Brumitt, Heiderscheit, Manske, Niemuth, \& Rauh, 2013). Actualmente son diversas las pruebas que detectan disfunciones músculo esqueléticas que se asocian a riesgo de lesión, ejemplo de ellas son la Y balance test (YBT) y el Lateral Step Down (LSD). La primera de ellas ha demostrado ser válida y fiable para evaluar el equilibrio dinámico en distintas poblaciones, y se ha utilizado para detectar alteraciones en la extremidad inferior o como predictor de riesgo de lesión (Chimera, Smith, \& Warren, 2015; Plisky, Rauh, Kaminski, \& Underwood, 2006). La utilidad para predecir riesgos de lesión del YBT ha sido evaluada en distintos tipos de lesiones como esguinces (Hoch \& McKeon, 2011; McCann, Crossett, Terada, Kosik, Bolding \& Gribble, 2017), dolor lumbar crónico (Hooper, James, Brismée, Rogers, Gilbert, Browne et al., 2016), lesiones de ligamento cruzado anterior (Cervenka, Decker, Ricard, Beaty, \& Ruhde, 2018). En el caso de LSD evalúa el control neuromuscular durante una tarea de descenso lateral de una extremidad desde un cajón, y sus resultados han demostrado ser indicadores de rangos de movimiento limitados o debilidad en los músculos de la cadera o tronco (Burnham, Yonz, Robertson, McKinley, Wilson, Johnson et al., 2016; Rabin \& Kozol, 2010), características que 
predisponen a un mayor riesgo de lesión. Un estudio que analizó el LSD en mujeres sanas con distinta calidad de movimiento, encontró que quienes presentaban una calidad moderada de movimiento muestran una dorsiflexión de tobillo limitada (Rabin \& Kozol, 2010). Burnhanm et al. (2016), analizó la calidad de movimiento durante la tarea de descenso de un cajón a una pierna, encontró que la fuerza del tronco y la cadera fue un predictor del control de movimiento durante la tarea evaluada.

Conocer cuáles son las características de los corredores recreativos que pueden aumentar el riesgo de lesión, puede ser una estrategia para mantener la adherencia y seguridad en la práctica deportiva en la población, disminuyendo de esta manera los índices de inactividad física y sedentarismo del país. Es por ello que el objetivo de este estudio es describir el equilibrio dinámico y control neuromuscular asociado a riesgo de lesión en corredores recreativos de la comuna de Chillán.

\section{METODOLOGÍA}

El estudio tiene un diseño no experimental, transeccional descriptivo. La muestra corresponde a 61 corredores aficionados entre 18 y 56 años de edad, 39 hombres $(63,9 \%)$ y 22 mujeres $(36,1 \%)$ elegidos por criterios de inclusión y exclusión.

Los criterios de inclusión fueron los siguientes: (I) Ser corredor aficionado de la ciudad de Chillán; (II) Pertenecer al grupo de corredores que participa en carreras entre $5 \mathrm{~km}$ y $12 \mathrm{~km}$; (III) Tener sobre 18 años de edad; (IV) Firmar consentimiento informado. Los criterios de exclusión fueron: (I) Mujeres en condición de embarazo; (II) Registrar alguna lesión en los últimos 6 meses; (III) Presentar alguna lesión actual diagnosticada por un especialista.

Los sujetos que aceptaron participar voluntariamente fueron informados sobre los objetivos del estudio y firmaron un consentimiento informado, cumpliendo con las recomendaciones del protocolo de Helsinki. Todos los participantes completaron un cuestionario con información sobre sexo, edad, peso, talla, categoría de competencia, historial de lesiones, pierna dominante. Luego fueron instruidos en la realización de los test funcionales en el siguiente orden: (1) Y Balance Test; (2) Lateral Step Down.

\section{Y Balance Test (YBT)}

El YBT es una prueba que evalúa el equilibrio dinámico y es utilizada para predecir el riesgo de lesión en las extremidades inferiores. Cada corredor recibió la explicación y ejemplificación de la forma en que se realiza el test según el protocolo descrito anteriormente en Plisky et al. (2006). Cada participante recibió una explicación verbal y una demostración visual de la ejecución del test. Para la evaluación se ubicó en posición unipodal con los pies descalzos sobre una plataforma de apoyo que une tres piezas de tubería en las direcciones anterior, posteromedial y posterolateral, en la que debía empujar con su extremidad libre un indicador de alcance que se deslizaba por la tubería. Cada sujeto recibió 6 ensayos de prueba y luego se registraron 3 intentos continuos en cada dirección (anterior, posterolateral y posteromedial), siendo descartado si perdía la posición unilateral, no lograba mantener la posición con el indicador de alcance, utilizaba el indicador de alcance para el soporte de la postura, o no lograba volver a la posición de inicio bajo control. Se registró la distancia en centímetros y normalizada ([Distancia alcanzada/longitud de la pierna $\left.]^{\star} 100\right)$. La medida global del rendimiento en la prueba se obtuvo promediando la distancia normalizada en cada dirección (Gribble, Hertel, \& Plisky, 2012).

La longitud de la pierna se midió en centímetros desde la espina iliaca anterosuperior hasta la porción más distal del maleolo medial.

\section{Lateral Step Down (LSD)}

Para evaluar el control neuromuscular se utilizó el LSD, test que mide la calidad del movimiento mientras realiza un descenso lateral de un cajón de 20 centímetros (Mostaed, Werner, \& Barrios, 2018). Los corredores realizaron la prueba unilateralmente de manera aleatoria 
para cada extremidad. Previo a la evaluación se demostró la acción de la prueba descrita por Piva, Fitzgerald, Irrgang, Jones, Hando, Browder et al. (2006). Cada sujeto evaluado, se ubicó en el borde del cajón en posición unipodal, con las manos en la cadera y rodilla recta, luego realiza una flexión de rodilla hasta que la pierna contralateral toma contacto suavemente con el piso para recuperar nuevamente su posición inicial. Se realizaron seis intentos excluyendo la primera y la última para el análisis. La evaluación fue evaluada mediante un registro visual ubicado a 3 metros del cajón alineado con el borde del cajón con la extremidad evaluada.

Para valorar la ejecución de la prueba se utilizaron 5 criterios:

Estrategia del brazo: si utiliza sus brazos para recuperar el equilibrio durante la ejecución se agrega 1 punto; movimiento del tronco: si durante la ejecución el tronco se inclina hacia uno de los lados se agrega 1 punto; plano de la pelvis: si la pevis se rota, o se eleva más de un lado en relación con el otro, se agrega 1 punto; posición de la rodilla: si la rodilla se desvía medialmente y la tuberosidad de la tibia cruza una línea vertical imaginaria sobre el segundo dedo del pie, se suma 1 punto o, si la rodilla cruza una línea imaginaria sobre el borde medial del pie, se agrega 2 puntos; posición unipodal: si la extremidad evaluada se vuelve inestable oscilando de un lado a otro, se agrega 1 punto.

\section{Análisis de la Información}

Para el tratamiento de datos se utilizó un valor de $\mathrm{p} \leq 0,05$. Todos los análisis estadísticos se efectuaron con el programa IBM SPSS en su versión 21, Para los análisis estadísticos los participantes fueron categorizados por grupos etarios: $<20$ años; $>20$ y $<30$ años; $>30$ a $<40$ años; y $>40$ año. Se utilizó estadística descriptiva para cada una de las variables tanto para el total de la muestra y para los subgrupos formados según rango de edad. Se realizó la prueba de Kolmogorov- Smirnov, para la distribución normal de la muestra, para luego realizar el análisis ANOVA de dos vías (4 grupos) con homogeneidad de varianza para comparar si existían diferencias significativas entre los grupos etarios.

\section{RESULTADOS}

Se evaluaron un total de 61 personas con una edad promedio de $31 \pm 22$ años 39 hombres $(63,9 \%)$ y 22 mujeres $(36,1 \%)$. Las características biotipológicas de la población incluida muestran diferencias significativas entre los grupos etarios para longitud de pierna, IMC, peso y edad $(\mathrm{p}<0,01)$. (Ver Tabla 1$)$.

Tabla 1

Características biotipológica de la población evaluada según rango de edad.

\begin{tabular}{ccccccc}
\hline & Total & $<20$ & $20<30$ & $30<40$ & $>40$ & $\mathrm{P}$ \\
\hline $\mathrm{N}$ & 61 & 23 & 21 & 8 & 9 & \\
Edad & $26,3 \pm 11,5$ & $16,6 \pm 1,4$ & $24,1 \pm 2,6$ & $34,5 \pm 2,7$ & $49,1 \pm 5,8$ & 0,001 \\
Peso & $66,1 \pm 11,4$ & $62,9 \pm 10,7$ & $63,4 \pm 11,9$ & $73,8 \pm 8,5$ & $73,6 \pm 8,4$ & 0,01 \\
Talla & $167,8 \pm 10,2$ & $169,7 \pm 11$ & $164,7 \pm 9,2$ & $170,9 \pm 10,1$ & $167,4 \pm 9,8$ & 0,329 \\
IMC & $23,4 \pm 2,8$ & $21,7 \pm 2,0$ & $23,2 \pm 2,5$ & $25,3 \pm 2,7$ & $26,2 \pm 1,7$ & 0,001 \\
LP & $93,2 \pm 6,2$ & $95,1 \pm 6,5$ & $91,6 \pm 6,2$ & $93,2 \pm 5,4$ & $92,4 \pm 6,0$ & 0,001 \\
\hline
\end{tabular}

IMC = Índice de Masa Corporal, LP = Longitud de la Pierna

Los resultados encontrados en la evaluación del Y-balance test son presentados en la Tabla 2 en función del alcance total y normalizadas para las direcciones anterior, posterolateral, posteromedial y asimetría para la pierna dominante y no dominante. La comparación entre los grupos etarios no muestra diferencias significativas entre ellos $(p>0,05)$. 
Tabla 2

Longitudes normalizadas y asimetría en Y-Balance Test.

\begin{tabular}{lcccccc}
\hline & Total & $<20$ & $20<30$ & $30<40$ & $>40$ & P \\
\hline Alcance Anterior & & & & & & \\
PD (\%) & $78,6 \pm 10,2$ & $75,5 \pm 7,7$ & $82,9 \pm 10,2$ & $76,3 \pm 10,2$ & $78,6 \pm 13,6$ & 0,089 \\
PND (\%) & $77,4 \pm 8,8$ & $74,9 \pm 7,4$ & $81,0 \pm 9,8$ & $77,0 \pm 10,2$ & $73,4 \pm 5,5$ & 0,061 \\
Asimetría (cm) & $4,4 \pm 4,2$ & $3,8 \pm 3,1$ & $4,4 \pm 4,0$ & $4,2 \pm 2,9$ & $6,2 \pm 7,5$ & 0,571 \\
Alcance Postero-Lateral & & & & & & \\
PD (\%) & $93,4 \pm 8,9$ & $94,1 \pm 8,9$ & $91,4 \pm 7,1$ & $95,6 \pm 9,8$ & $94,1 \pm 12,1$ & 0,626 \\
PND (\%) & $93,9 \pm 9,7$ & $94,5 \pm 8,0$ & $92,7 \pm 11,1$ & $94,5 \pm 10,5$ & $94,4 \pm 10,9$ & 0,931 \\
Asimetría (cm) & $6,3 \pm 4,2$ & $6,9 \pm 5,2$ & $6,4 \pm 3,7$ & $5,4 \pm 3,6$ & $5,7 \pm 2,7$ & 0,802 \\
Alcance Postero Medial & & & & & & \\
PD (\%) & $88,8 \pm 9,2$ & $88,5 \pm 8,1$ & $87,9 \pm 9,8$ & $87,1 \pm 11,3$ & $93,2 \pm 8,6$ & 0,472 \\
PND (\%) & $89,5 \pm 8,5$ & $89,9 \pm 7,6$ & $87,4 \pm 7,5$ & $91,1 \pm 10,8$ & $92,1 \pm 10,6$ & 0,493 \\
Asimetría (cm) & $5,4 \pm 3,8$ & $5,4 \pm 4,0$ & $5,8 \pm 3,7$ & $5,6 \pm 4,3$ & $4,1 \pm 3,1$ & 0,720 \\
Puntaje Compuesto & & & & & & \\
PD (\%) & $87,0 \pm 7,5$ & $86,0 \pm 7,2$ & $87,9 \pm 6,7$ & $86,2 \pm 7,5$ & $88,1 \pm 10,7$ & 0,807 \\
PND (\%) & $86,9 \pm 6,8$ & $86,7 \pm 6,7$ & $86,6 \pm 7,0$ & $87,7 \pm 6,3$ & $87,2 \pm 8,1$ & 0,985 \\
Total (\%) & $87,0 \pm 9,5$ & $86,2 \pm 8,0$ & $87,2 \pm 9,3$ & $86,9 \pm 10,5$ & $87,6 \pm 10,2$ & 0,992 \\
\hline
\end{tabular}

La Tabla 3 muestra los resultados en LSD con las medias y desviación estándar de los puntajes globales logrados en el LSD conside- rando la pierna dominante y no dominante, sin encontrar diferencias significativas entre los distintos grupos etarios de la muestra.

Tabla 3

Calidad del movimiento según Lateral Step Down.

\begin{tabular}{lcccccc}
\hline & Total & $<20$ & $20<30$ & $30<40$ & $>40$ & P \\
\hline LSD_PD (cm) & $2,9 \pm 1,4$ & $2,7 \pm 1,5$ & $3,0 \pm 1,3$ & $2,8 \pm 1,8$ & $3,0 \pm 1,3$ & 0,845 \\
LSD_PND (cm) & $2,8 \pm 1,3$ & $2,7 \pm 1,4$ & $2,9 \pm 1,1$ & $3,4 \pm 0,7$ & $2,7 \pm 1,7$ & 0,636 \\
\hline
\end{tabular}

Los resultados de las estrategias de movimiento empleados para la ejecución del test son presentados en la Figura 1. 
Test Lateral Step Down (LSD) según estrategia utilizada

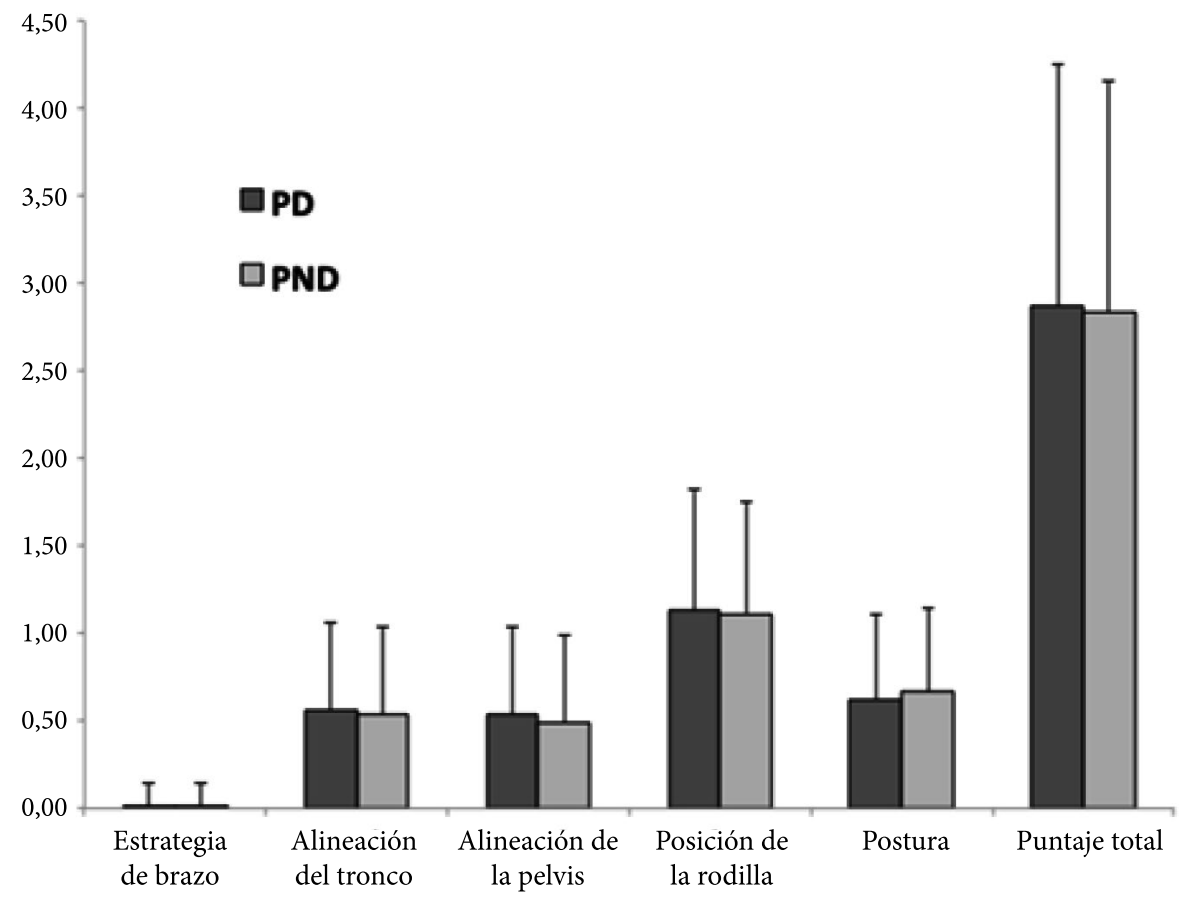

${ }^{\star}$ PD: Pierna dominante, PND: Pierna no dominante.

Figura 1. Resultados en LSD según estrategia utilizada.

De acuerdo a los resultados, no existen diferencias entre grupos etarios para ninguna de las pruebas realizadas, la calidad de movimiento es moderada (según el LSD), y un equilibrio dinámico asimétrico al comparar las extremidades dominantes y no dominante.

\section{DISCUSIÓN}

El propósito de este estudio fue describir el equilibrio dinámico y control neuromuscular asociado a riesgo de lesión en corredores recreativos de la comuna de Chillán. Los resultados de este estudio muestran que los corredores recreativos poseen un equilibrio dinámico asimétrico al comparar las extremidades dominantes y no dominante y la calidad de movimiento evaluada por el test LSD es moderada.

Los resultados para YBT muestra que la puntuación compuesta fue de $87,0 \pm 7,5$ y $86,9 \pm 6,8$ para la pierna dominante y no dominante. Un análisis según grupo etario no mostró diferencias significativas entre ellos, lo que no coincide con estudios que han utilizado el mismo instrumento y que analizaron la influencia de la edad sobre el rendimiento en YBT (Teyhen, Riebel, McArthur, Savini, Jones, Goffar et al., 2014), sin embargo, la población incluida en ese estudio corresponde a personal militar y sus valores estaban por sobre los encontrados en nuestra población. Estudios que ofrecen valores de referencia varían en cuanto a la población estudiada, encontrando en general que cuando se evalúan a grupos activos físicamente presentan mejores resultados en comparación a población en general (Alnahdi, Alderaa, Aldali, \& Alsobayel, 2015; Engquist, Smith, Chimera, \& Warren, 2015; Teyhen et al., 2014). Un estudio realizado en Arabia Saudita presentó valores de referencia para población sana en el alcance anterior ( $70 \%$ de la longitud de la pierna), posteromedial (93\% de la longitud de la pierna), y posterolateral (93\% de la longitud de la pierna), finalmente el puntaje compuesto de 
ambas extremidades considera la normalidad en $85 \%$ de la longitud de la pierna (Alnahdi et al., 2015). Por otra parte Engquist et al. (2015), comparó los alcances en el YBT en las tres dirección entre una población de universitarios de distintos deportes versus universitarios que no practican deporte, encontrando que el grupo de deportistas presentó mejores resultados para el alcance anterior $(74,7 \pm 0,6 \mathrm{v} / \mathrm{s} 71,7$ $\pm 0,7)$, posteromedial $(116,4 \pm 0,8 \mathrm{v} / \mathrm{s} 110,7$ $\pm 1,1)$ y posterolateral $(114,2 \pm 0,8 \mathrm{v} / \mathrm{s} 108,6$ $\pm 1,17)$ estas diferencias seguramente responde a las demandas asociadas a los deportes que exigen una mejor integración sensorial para responder de manera más eficaz a las distintas tareas motrices. Al comparar con la muestra podemos encontrar resultados cercanos a la población sana (Alnahdi et al., 2015), sin embargo, al comparar con población que realiza algún deporte encontramos distancias de alcances menores en posteromedial y posterolateral, pero mayores en alcance anterior.

$\mathrm{Al}$ analizar los resultados en el YBT asociados al riesgo de lesión encontramos que los corredores recreativos incluidos en este estudio, se encuentran por debajo de lo recomendado, mostrando un equilibrio dinámico asimétrico al comparar ambas extremidades y puntajes similares a la población sana, pero por debajo de la población deportista. Al considerar el puntaje compuesto se ha encontrado que mujeres basquetbolistas con un $94 \%$ en el puntaje compuesto poseen un $6,5 \%$ más posibilidades de lesionarse la extremidad inferior (Plisky et al., 2006) y en jugadores de fútbol americano que presentan un $89,6 \%$ también presentan más riesgo de lesión (Butler, Lehr, Fink, Kiesel, \& Plisky, 2013), para nuestra población encontramos que el puntaje compuesto fue de $87,0 \pm 9,5$ lo que implicaría un mayor riesgo de lesión, sin embargo, no encontramos investigaciones que utilizaran corredores recreativos para determinar un punto de corte para el riesgo de lesión, por lo que es necesario tomar con cautela esta interpretación. Además la asimetría anterior también ha sido relacionada con un aumento en el riesgo de lesión considerando que cuando es mayor a 4 centímetros el riesgo aumenta (Butler et al., 2013; Plisky et al., 2006), característica que también es encontrada en nuestra muestra.
Los resultados en el test LSD muestran que la calidad de movimiento durante un ejercicio de reducción lateral en un cajón de 20 centímetros es moderada (puntaje: 2,9 $\pm 1,4$ para la pierna dominante y $2,8 \pm 1,3$ para la pierna no dominante), siendo importante la consistencia del valgo de rodilla acentuado durante la ejecución del test. Estos resultados tampoco muestran diferencias significativas entre los grupos etarios o pierna evaluada, Una mala calidad de movimiento se ha encontrado en sujetos luego de la reconstrucción de ligamento cruzado anterior, inestabilidad crónica de tobillo, dolor femoropatelar, y otro tipo de lesiones de extremidad inferior. Luego de una reconstrucción de ligamento cruzado anterior una calidad moderada de movimiento se asoció a un aumento en la aducción de cadera y rotación interna de cadera, lo que podría sugerir que es necesario un fortalecimiento de la musculatura abductora y rotadora de cadera, así como también de la musculatura extensora de rodilla para mejorar el control neuromuscular (Mostaed et al., 2018). Similares resultados se encontraron al evaluar sujetos con dolor patelofemoral, mostrando que aquellas que presentaban mala calidad del movimiento poseían un menor rango de movimiento en la dorsiflexión de tobillo y la debilidad en la musculatura de la cadera parece explicar el déficit en el control de movimiento en aquellos que tenían un buen rango de dorsiflexión de tobillo (Rabin, Kozol, \& Finestone, 2014), del mismo modo sujetos con inestabilidad crónica de tobillo, han mostrado tener una menor dorsiflexión de tobillo siendo relacionada a una mala calidad de movimiento en el LSD (Grindstaff, Dolan, \& Morton, 2017).

En general los corredores principiantes muestran una prevalencia de lesiones distinta a corredores experimentados, encontrando una alta incidencia le lesiones leves como de esguinces de tobillo, ampollas y abrasiones (de Araujo et al., 2015). Otros estudios han encontrado que el síndrome de estrés tibial, el dolor patelofemoral, lesiones meniscales y tendinopatia de aquiles sueles ser recurrentes en corredores novatos (Nielsen, Rønnow, Rasmussen, \& Lind, 2014). Las lesiones causan un aumento en el ausentismo a entrenamientos el cual puede alcanzar hasta el 78\% 
de los corredores que se lesionan y una visita a profesionales sanitarios que alcanza el $51 \%$ (Smits, Huisstede, Verhagen, Van der Worp, Kluitenberg, Van Middelkoop et al., 2016), por lo tanto las evaluaciones pre-participativas deberían incluir una evaluación funcional de las extremidades inferiores para reducir la tasa de lesiones y el ausentismo a entrenamiento en corredores recreativos (Magrum \& Wilder, 2010; Vincent, Herman, Lear-Barnes, Barnes, Chen, Greenberg et al., 2014).

\section{CONCLUSIÓN}

De acuerdo a los resultados encontrados en el presente estudio los corredores recreativos tienen un moderado control neuromuscular evaluado mediante la prueba de LSD y un equilibrio dinámico evaluado por YBT similar a la población adulta sana pero más bajo que los reportados por población deportista, además presenta una asimetría en el alcance anterior $(>4 \mathrm{~cm})$ que es considerado un factor de riesgo para lesiones en extremidad inferior.

Es importante que las personas que comienzan a participar de corridas urbanas, se evalúen por profesionales de la actividad física, que puedan realizar evaluaciones pre-participativas que permitan desarrollar un programa de fortalecimiento muscular que disminuya el riesgo de lesiones.

\section{REFERENCIAS BIBLIOGRÁFICAS}

Alnahdi, A. H., Alderaa, A. A., Aldali, A. Z., \& Alsobayel, H. (2015). Reference values for the Y Balance Test and the lower extremity functional scale in young healthy adults. Journal of Physical Therapy Science, 27(12), 3917-3921. https://doi.org/10.1589/jpts.27.3917

Brumitt, J., Heiderscheit, B. C., Manske, R. C., Niemuth, P. E., Mattocks, A., \& Rauh, M. J. (2018). Preseason Functional Test Scores Are Associated With Future Sports Injury in Female Collegiate Athletes. Journal of strength and conditioning research, 32(6), 1692-1701. https://doi.org/10.1519/ ISC.0000000000002243

Brumitt, J., Heiderscheit, B. C., Manske, R. C., Niemuth, P. E., \& Rauh, M. J. (2013). Lower extremity functional tests and risk of injury in division iii collegiate athletes. International journal of sports physical therapy, 8(3), 216-227. Recuperado de http://www.ncbi.nlm.nih. gov/pubmed/23772338

Buist, I., Bredeweg, S. W., Lemmink, K. A., Pepping, G.J., Zwerver, J., van Mechelen, W., \& Diercks, R. L. (2007). The GRONORUN study: is a graded training program for novice runners effective in preventing running related injuries? Design of a Randomized Controlled Trial. BMC Musculoskeletal Disorders, 8(1), 24. https://doi.org/10.1186/14712474-8-24

Burnham, J. M., Yonz, M. C., Robertson, K. E., McKinley, R., Wilson, B. R., Johnson, D. L., ... Noehren, B. (2016). Relationship of Hip and Trunk Muscle Function with Single Leg Step-Down Performance: Implications for Return to Play Screening and Rehabilitation. Physical Therapy in Sport, 22, 66-73. https://doi. org/10.1016/J.PTSP.2016.05.007 
Butler, R. J., Lehr, M. E., Fink, M. L., Kiesel, K. B., \& Plisky, P. J. (2013). Dynamic Balance Performance and Noncontact Lower Extremity Injury in College Football Players. Sports Health: A Multidisciplinary Approach, 5(5), 417-422. https:// doi.org/10.1177/1941738113498703

Cervenka, J., Decker, M., Ricard, M., Beaty, J., \& Ruhde, L. (2018). Strength and Stability Analysis of Rehabilitated Anterior Cruciate Ligament Individuals. International Journal of Exercise Science, 11(1), 817-826. Recuperado de https:// digitalcommons.wku.edu/ijes/vol11/ iss $1 / 10$

Chimera, N. J., Smith, C. A., \& Warren, M. (2015). Injury History, Sex, and Performance on the Functional Movement Screen and Y Balance Test. Journal of Athletic Training, 50(5), 475-485. https://doi.org/10.4085/1062-6050$\underline{49.6 .02}$

Clausen, J. S. R., Marott, J. L., Holtermann, A., Gyntelberg, F., \& Jensen, M. T. (2018). Midlife Cardiorespiratory Fitness and the Long-Term Risk of Mortality: 46 Years of Follow-Up. Journal of the American College of Cardiology, 72(9), 987-995. https://doi.org/10.1016/I. JACC.2018.06.045

Conley, K. M., Bolin, D. J., Carek, P. J., Konin, J. G., Neal, T. L., \& Violette, D. (2014). National Athletic Trainers' Association Position Statement: Preparticipation Physical Examinations and Disqualifying Conditions. Journal of Athletic Training, 49(1), 102-120. https://doi. org/10.4085/1062-6050-48.6.05

Crump, C., Sundquist, J., Winkleby, M. A., \& Sundquist, K. (2017). Interactive Effects of Aerobic Fitness, Strength, and Obesity on Mortality in Men. American journal of preventive medicine, 52(3), 353-361. https://doi.org/10.1016/j. amepre.2016.10.002 de Araujo, M. K., Baeza, R. M., Zalada, S. R. B., Alves, P. B. R., \& de Mattos, C. A. (2015). Injuries among amateur runners. Revista Brasileira de Ortopedia (English Edition), 50(5), 537-540. https://doi. org/10.1016/J.RBOE.2015.08.012

Engquist, K. D., Smith, C. A., Chimera, N. J., \& Warren, M. (2015). Performance Comparison of Student-Athletes and General College Students on the Functional Movement Screen and the Y Balance Test. Journal of Strength and Conditioning Research, 29(8), 2296-2303. https://doi.org/10.1519/ ISC.0000000000000906

Gribble, P. A., Hertel, J., \& Plisky, P. (2012). Using the Star Excursion Balance Test to Assess Dynamic Postural-Control Deficits and Outcomes in Lower Extremity Injury: A Literature and Systematic Review. Journal of Athletic Training, 47(3), 339-357. https://doi. org/10.4085/1062-6050-47.3.08

Grindstaff, T. L., Dolan, N., \& Morton, S. K. (2017). Ankle dorsiflexion range of motion influences Lateral Step Down Test scores in individuals with chronic ankle instability. Physical Therapy in Sport, 23, 75-81. https://doi. org/10.1016/J.PTSP.2016.07.008

Guthold, R., Stevens, G. A., Riley, L. M., \& Bull, F. C. (2018). Worldwide trends in insufficient physical activity from 2001 to 2016: a pooled analysis of 358 population-based surveys with $1.9 \mathrm{mi}$ llion participants. The Lancet Global Health, 6(10), e1077-e1086. https://doi. org/10.1016/S2214-109X(18)30357-7

Hoch, M. C., \& McKeon, P. O. (2011). Joint mobilization improves spatiotemporal postural control and range of motion in those with chronic ankle instability. Journal of Orthopaedic Research, 29(3), 326-332. https://doi.org/10.1002/ jor. 21256 
Hooper, T. L., James, C. R., Brismée, J. M., Rogers, T. J., Gilbert, K. K., Browne, K. L., \& Sizer, P. S. (2016). Dynamic balance as measured by the Y-Balance Test is reduced in individuals with low back pain: A cross-sectional comparative study. Physical Therapy in Sport, 22, 29-34. https://doi.org/10.1016/J. PTSP.2016.04.006

Kluitenberg, B., van Middelkoop, M., Diercks, R., \& van der Worp, H. (2015). What are the Differences in Injury Proportions Between Different Populations of Runners? A Systematic Review and Meta-Analysis. Sports Medicine, 45(8), 1143-1161. https://doi.org/10.1007/ s40279-015-0331-x

Magrum, E., \& Wilder, R. P. (2010). Evaluation of the injured runner. Clinics in sports medicine, 29(3), 331-345. https://doi. org/10.1016/j.csm.2010.03.009

McCann, R. S., Crossett, I. D., Terada, M., Kosik, K. B., Bolding, B. A., \& Gribble, P. A. (2017). Hip strength and star excursion balance test deficits of patients with chronic ankle instability. Journal of science and medicine in sport, 20(11), 992-996. $\quad$ https://doi.org/10.1016/j. jsams.2017.05.005

Mostaed, M. F., Werner, D. M., \& Barrios, J. A. (2018). 2d and 3d kinematics during lateral step-down testing in individuals with anterior cruciate ligament reconstruction. International Journal of Sports Physical Therapy, 13(1), 77-85. https:// doi.org/10.26603/ijspt20180077

Nielsen, R. O., Rønnow, L., Rasmussen, S., \& Lind, M. (2014). A Prospective Study on Time to Recovery in 254 Injured Novice Runners. PLoS ONE, 9(6), e99877. https://doi.org/10.1371/journal.pone.0099877
Nyberg, S. T., Batty, G. D., Pentti, J., Virtanen, M., Alfredsson, L., Fransson, E. I., ... Kivimäki, M. (2018). Obesity and loss of disease-free years owing to major non-communicable diseases: a multicohort study. The Lancet. Public health, 3(10), e490-e497. https://doi. org/10.1016/S2468-2667(18)30139-7

Piva, S. R., Fitzgerald, K., Irrgang, J. J., Jones, S., Hando, B. R., Browder, D. A., \& Childs, J. D. (2006). Reliability of measures of impairments associated with patellofemoral pain syndrome. BMC Musculoskeletal Disorders, 7(1), 33. https://doi. org/10.1186/1471-2474-7-33

Plisky, P. J., Rauh, M. J., Kaminski, T. W., \& Underwood, F. B. (2006). Star Excursion Balance Test as a Predictor of Lower Extremity Injury in High School Basketball Players. Journal of Orthopaedic \& Sports Physical Therapy, 36(12), 911-919. https://doi.org/10.2519/ jospt.2006.2244

Rabin, A., \& Kozol, Z. (2010). Measures of Range of Motion and Strength Among Healthy Women With Differing Quality of Lower Extremity Movement During the Lateral Step-Down Test. Journal of Orthopaedic \& Sports Physical Therapy, 40(12), 792-800. https://doi. org/10.2519/jospt.2010.3424

Rabin, A., Kozol, Z., \& Finestone, A. S. (2014). Limited ankle dorsiflexion increases the risk for mid-portion Achilles tendinopathy in infantry recruits: a prospective cohort study. Journal of Foot and Ankle Research, 7(1), 48. https://doi. org/10.1186/s13047-014-0048-3

Sandoval Vilches, P., \& García Pacheco, I. (2014). Cultura deportiva en Chile: desarrollo histórico, institucionalidad actual e implicancias para la política pública. Polis. Revista Latinoamericana, (39). Recuperado de https://journals. openedition.org/polis/10583 
Smits, D.W., Huisstede, B., Verhagen, E., van der Worp, H., Kluitenberg, B., van Middelkoop, M., ... Backx, F. (2016). Short-Term Absenteeism and Health Care Utilization Due to Lower Extremity Injuries Among Novice Runners: A Prospective Cohort Study. Clinical journal of sport medicine: official journal of the Canadian Academy of Sport Medicine, 26(6), 502-509. https://doi. org/10.1097/ISM.0000000000000287

Teyhen, D. S., Riebel, M. A., McArthur, D. R., Savini, M., Jones, M. J., Goffar, S. L., ... Plisky, P. J. (2014). Normative Data and the Influence of Age and Gender on Power, Balance, Flexibility, and Functional Movement in Healthy Service Members. Military Medicine, 179(4), 413-420. https://doi. org/10.7205/MILMED-D-13-00362

Vincent, H. K., Herman, D. C., Lear-Barnes, L., Barnes, R., Chen, C., Greenberg, S., \& Vincent, K. R. (2014). Setting Standards for Medically-Based Running Analysis. Current sports medicine reports, 13(4), 275. https://doi. org/10.1249/JSR.0000000000000071

\section{Dirección para correspondencia}

Nicolás Alexander Gómez-Álvarez

Universidad Adventista de Chile.

Chillán, Chile.

Contacto:

nicolasgomez@unach.cl

Recibido: 27-12-2018

Aceptado: 14-06-2019 